\title{
The Prevalence of Undiagnosed Depression in Patients with Spinal Pathology
}

\author{
Menendez JY1, Dawkins RL ${ }^{1 *}$, Walters BC', \\ Shelton RC ${ }^{2}$, Markert J $\mathrm{M}^{\mathbf{1}}$ and Pritchard PR $^{1}$ \\ ${ }^{1}$ Department of Neurosurgery, University of Alabama at \\ Birmingham, USA \\ ${ }^{2}$ Department of Psychiatry, University of Alabama at \\ Birmingham, USA \\ *Correspondling author: Dawkins RL, Department of \\ Neurosurgery, Vanderbilt University, T4224 MCN, 1161 \\ 21st Avenue South Nashville, TN 37232-2380, USA
}

Received: April 04, 2018; Accepted: May 07, 2018; Published: May 15, 2018

\begin{abstract}
Study Design: Cross-sectional study

Objectives: Depression is a comorbid condition with significant effects on patients with spinal pathology, and is often undiagnosed. The objective was to define the prevalence of undiagnosed depression in our population of patients with neck or back pain using a standard screening tool.

Methods: Patients presenting to a neurosurgery clinic at our institution for initial evaluation of neck or back pain were screened for depression with the revised Center for Epidemiologic Studies Depression (CES-D) scale.

Results: Of the 76 patients who completed screening, 20 patients $(26.3 \%)$ had scores that were suspicious for a diagnosis of depression, and the suspicion was newly discovered for all 20 patients (26.3\%). There were 20 patients (26.3\%) with scores that were consistent with a diagnosis of depression, and the diagnosis was newly discovered for 17 patients (22.4\%). Thus, a total of 37 patients (48.7\%) had a suspicion for or diagnosis of depression that was previously undiagnosed.
\end{abstract}

Conclusion: Depression in patients with spinal pathology frequently goes undiagnosed, as demonstrated by the results of our cross sectional study. This contributes to the mounting evidence for the regular screening and treatment of depression in patients with spinal pathology.

Keywords: Depression; Mood disorder; Prevalence; Spine

\section{Abbreviations}

CES-D: Center for Epidemiologic Studies Depression screening tool; DSM-IV: Diagnostic and Statistical Manual of Mental Disorders, $4^{\text {th }}$ Edition

\section{Introduction}

Depression is a common disorder affecting $6.9 \%$ of the adult population in the United States [1]. It is a leading cause of lost wages and productivity and the cost of treating depression is rising [2]. Depression is frequently a co-morbid condition in patients with pathology of the spine and is more frequent in patients with higher pain scores for back and leg pain [3,4]. The negative impact of depression on the post-operative outcomes from spinal operations has been similarly well established $[5,6]$. Despite the clear association between depression and spine pathology, there is very little evidence of formalized screening for depression during the evaluation of patients for spinal surgery or an understanding of how frequently patients undergoing spinal surgery suffer from depression [7]. The purpose of this study is to define the prevalence of undiagnosed depression as a co-morbid condition among patients suffering from neck or back pain. This is a pilot study for future investigation into the associations between characteristics of our patients and depression, as well as the effects of depression treatment on patient outcomes.

\section{Materials and Methods}

\section{Study population}

Over a one month period, adult patients presenting to a neurosurgery outpatient clinic at our institution for initial evaluation of neck or back pain were screened for depression using the revised Center for Epidemiologic Studies Depression form (CES-D), a wellestablished tool for the initial diagnosis of depression (Figure 1) [8,9]. The CES-D has been utilized across a variety of clinical and research settings [10-12]. The patient population consisted of patients with a history of neck pain, back pain, and/or extremity symptoms that had been referred to neurosurgery clinic for further evaluation. The referring physician had obtained spinal imaging which was concerning for pathological findings that were thought to be associated with the patient's symptoms. We refer to these patients as neck/back pain patients. After obtaining approval from the University of Alabama Birmingham Institutional Review Board (IRB-150526002), a retrospective review of the results of the screening was undertaken. Participants were not required to provide informed consent.

\section{Data collection}

The CES-D was given to a representative sampling of patients presenting to neurosurgery clinics for evaluation of neck/back pain over a four-week period. Completion of the form was voluntary. A 
Briet Montal Well-Being Survoy

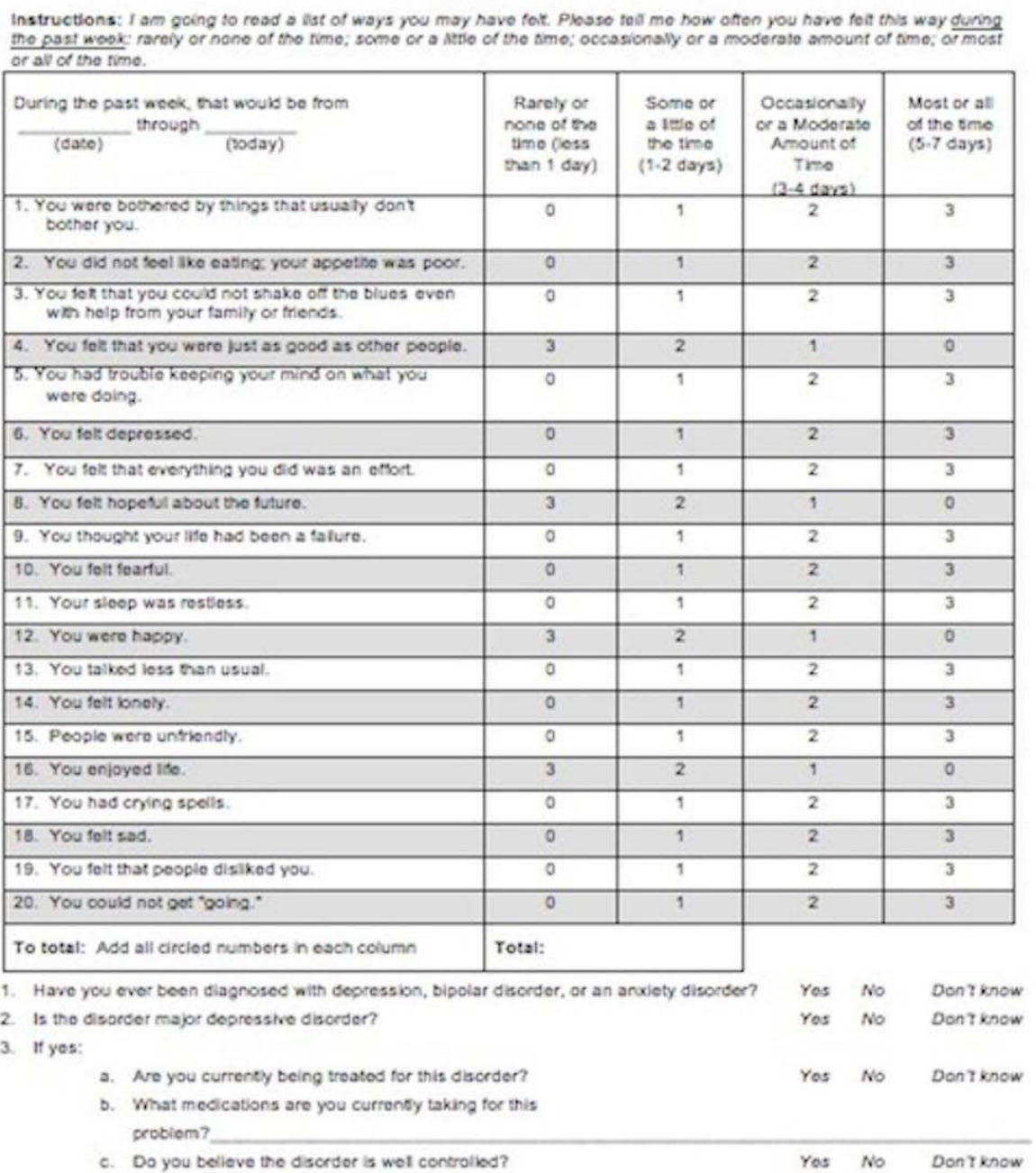

Figure 1: The Center for epidemiologic studies depression scale revised.

study author manually calculated the total score for each subject and recorded the answers to the final three questions to assess whether subjects carried a prior diagnosis of major depressive disorder. Per accepted interpretation of the CES-D [13], scores less than 10 were interpreted as not indicating depression, scores ranging from 10-15 were interpreted as suggestive of depression and scores greater than or equal to 16 were interpreted as diagnostic of depression.

\section{Results}

There were 76 patients newly assessed for neck/back pain in neurosurgical clinics that completed the CES-D screening tool during the study period. There were 20 patients (26.3\%) who scored $10-15$ on the CES-D, indicating a suspicion for depression. Another 20 patients (26.3\%) scored 16 or above on the CES-D, indicating a diagnosis of depression (Figure 2). Thus, a total of 40 patients $(52.6 \%$ ) had a suspicion for or diagnosis of depression.

Of the 20 patients who scored in the 10-15 range, all 20 (26.3\%) had no prior diagnosis of depression. Of the 20 subjects who scored 16 or greater on the CES-D, 17 (22.4\%) had no prior diagnosis of depression. Thus, a total of 37 patients (48.7\%) had a new suspicion for or diagnosis of depression (Figure 3).

Of the 76 patients who completed the screening tool, 19 stated that they have a prior diagnosis of a mood disorder, and 5 of those 19 patients stated that the mood disorder is major depressive disorder. Three of the 19 patients scored 16 or greater, and the other 16 patients scored $0-9$. Of the 19 patients who stated that they have a mood disorder, 13 patients (68\%) were receiving treatment. Of the 5 patients who stated they have a prior diagnosis of depression, $4(80 \%)$ were receiving treatment.

\section{Discussion}

A common goal amongst surgeons treating neck/back pain is the optimization of outcomes after surgery. Improvements in surgical technique and perioperative care are obviously important for the optimization of outcomes, but attention to patient comorbidities is also deserved. However, in order for the modification of a patient's comorbidity, such as depression, to be worth the expense of time and resources, a few conditions must be met.

First, it must be demonstrated that a significant percentage of patients with neck/back pain actually carry a diagnosis of depression. 


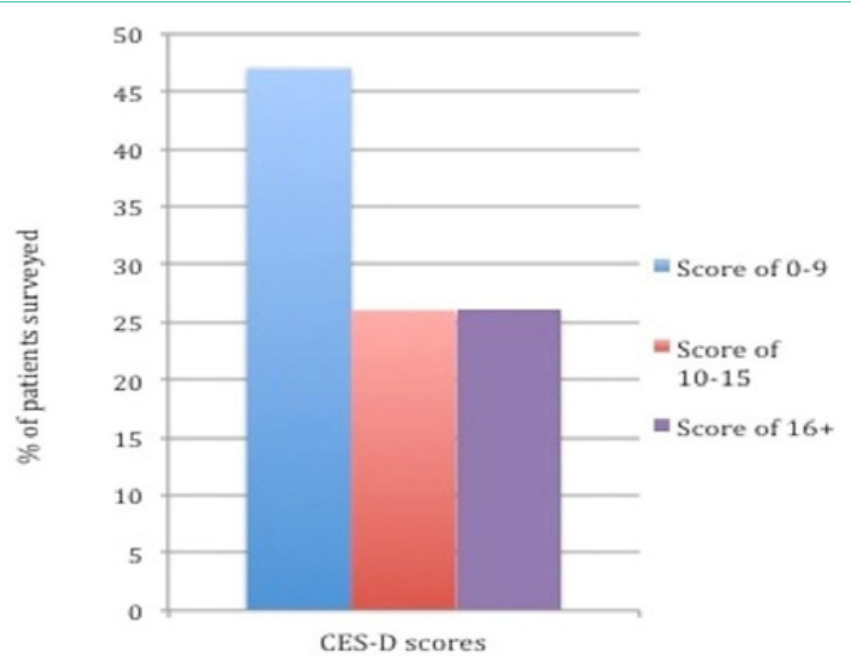

Figure 2: Graphic presentation of CES-D scores for all patients. $47.4 \%$ of patients scored 0-9, which indicates no depression. $26.3 \%$ of patients scored 10-15, which indicates a suspicion for a diagnosis of depression. $26.3 \%$ of patients scored $16+$, which is diagnostic of depression.

Furthermore, it must be clear that depression is associated with worse symptoms and worse outcomes after treatment. We already know that depression is a common condition affecting nearly $7 \%$ of the general population at any point in time [1] and that it is a leading cause of lost productivity and wages [2]. Studies have also shown that depression is a significant comorbidity in patient populations suffering from a variety of physical diseases, including diabetes mellitus, HIV/AIDS, cardiothoracic pathology, liver disease, and epilepsy [14-18]. Our disease of interest was neck/back pain, and thus the purpose of our study was to clarify the prevalence of depression among patients with neck/back pain, and more specifically, the prevalence of undiagnosed depression. Previous studies have used a variety of screening tools to look for evidence of depression in patients with neck/back pain, with a prevalence ranging from $21 \%$ to $56 \%[3,4]$. Our results are similar, indicating a much higher prevalence of depression $(26.3 \%)$ in patients presenting for evaluation of neck/back pain as compared to the general population, with another $26.3 \%$ having a suspicion of depression. Furthermore, by using the revised CES-D, we were able to elucidate the prevalence specifically of undiagnosed depression, which has not previously been examined in spine patients. We found that of all patients who scored in the range indicative of a diagnosis of depression, the overwhelming majority (85\%) were previously undiagnosed. The proportion of the entire study cohort with previously undiagnosed depression or concern for depression was also significant (48.7\%). Not only is depression affecting a significant portion of our patient population, but its presence, and the consequences, are going unnoticed. Depression is known to be associated with worse symptoms and functional status, increased pain and stress, higher use of narcotics and antidepressants, and overall dissatisfaction with life in patients with spinal pathology $[4,19]$. We also know that depression is associated with worse outcomes and patient satisfaction following spine surgery $[5,6,20]$.

We acknowledge that the effects of spinal pathology, including increased pain and decreased functional status, may contribute to increased rates of depression in this patient population. This presents

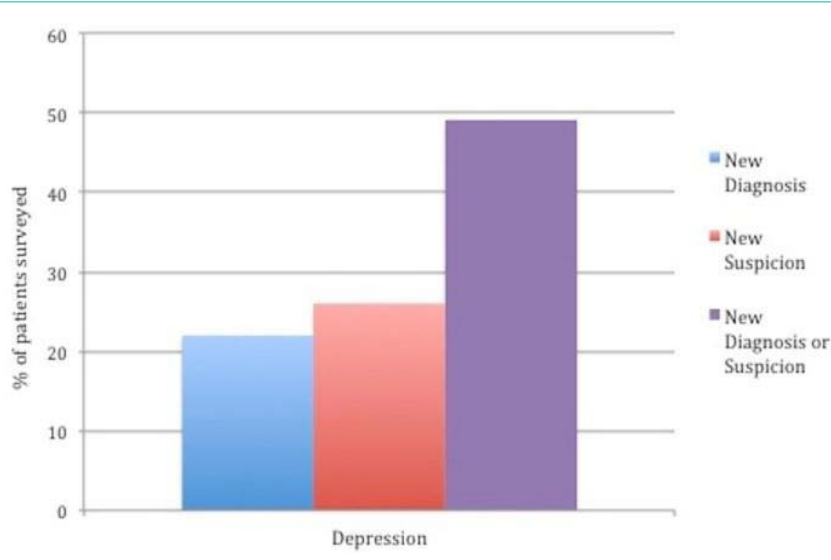

Figure 3: Graphic presentation of patients with a new suspicion or diagnosis of depression. Patients were categorized as having a new suspicion or diagnosis of depression based on the score from the CES-D survey in addition to the answers to the questions at the end of the survey.

a tempting option to simply treat the neck/back pain, in hopes that the improved symptoms will be followed by improved depression. However, the relationship is more complex, and the two disease entities are more interactive. In a study by Urban-Baez, surgery for spinal stenosis improved preoperative depression symptoms at the one-year follow-up. However, persistence of depressive symptoms after surgery correlated with a worse clinical outcome and a higher rate of unmet expectations [20]. Furthermore, in a study of patients with chronic low back pain, $54 \%$ of patients with a positive lifetime history of depression developed symptoms before the onset of back pain [21]. Clearly depression is not simply a consequence of the spinal disease for many in this patient population, and deserves its own treatment. We also acknowledge that it is difficult to distinguish between symptoms of pain and symptoms of depression, making it hard to tell if a patient with pain related to spine pathology also has depression. However, the CES-D can be of great assistance with this problem. The CES-D has been shown to be a sensitive tool for predicting depression, as diagnosed by the Diagnostic and Statistical Manual of Mental Disorders, $4^{\text {th }}$ Edition (DSM-IV) criteria, among patients with chronic pain [22].

There must be a reasonable method of screening for and treating depression. The CES-D is a self-report scale that was developed for use in epidemiological studies of depressive symptomatology in the general population [13]. We know that screening for depression with the CES-D is straightforward and the interpretation of this screening tool is well established [23]. A revised CES-D was created in 2004 in order to better reflect the nine primary symptoms of a major depressive episode as defined by the DSM-IV [8]. The revised version has also proven to be a valid measure of depression [9]. In our experience, the revised CES-D was easily completed by the patients and easily interpreted by the study authors. Furthermore, the low cost of administration is outweighed by the benefit of depression treatment. We know that depression is treatable and the effect of treatment on overall quality of life is positive and clear [24]. In our study, the majority (80\%) of those who already carried a diagnosis of depression were receiving treatment.

To warrant the involvement of a spine surgeon in the treatment 
of depression, it must be shown that adequate treatment results in improved outcomes after surgery for spinal pathology. By elucidating the prevalence of undiagnosed depression in our spine patient population, this study is serving as a pilot study for future investigations into the role depression plays in patients with spinal pathology, and how the treatment of depression affects their outcomes.

Given that depression has been shown to be more common in patients with neck/back pain and often goes undiagnosed, has been demonstrated to have a negative effect on outcomes, has a screening tool that is easily implemented and interpreted, and is known to be amenable to multi-modality therapy; it stands to reason that screening for depression in spine patients and treating newly diagnosed depression will improve overall quality of life as well as outcomes following spine surgery.

\section{Conclusion}

Depression is a commonly recognized co-morbid condition in patients with neck/back pain. The objective of this cross-sectional study was to define the prevalence of undiagnosed depression in our spine patients. The results indicate that the true prevalence of depression in patients with neck/back pain is higher than in the general population. For a significant portion of the patients, the depression has previously been undiagnosed. Screening for depression is relatively simple and should be carried out to identify patients with undiagnosed depression, as this co-morbid condition can negatively impact outcomes from intervention aimed at addressing neck/back pain

\section{References}

1. Major depression among adults. 2016.

2. Luppa M, Heinrich S, Angermeyer MC, König HH, Riedel-Heller SG. Costof-illness studies of depression: a systematic review. J Affect Disord 2007; 98: 29-43.

3. Dersh J, Gatchel RJ, Mayer T, Polatin P, Temple OR. Prevalence of psychiatric disorders in patients with chronic disabling occupational spinal disorders. Spine. 2006; 31: 1156-1162.

4. Sinikallio $\mathrm{S}$, Aalto $\mathrm{T}$, Airaksinen $\mathrm{O}$, Herno $\mathrm{A}$, Kröger $\mathrm{H}$, Savolainen $\mathrm{S}$, et al. Depression and associated factors in patients with lumbar spinal stenosis. Disabil Rehabil. 2006; 28: 415-422.

5. Menendez ME, Neuhaus V, Bot AG, Ring D, Cha TD. Psychiatric disorders and major spine surgery: epidemiology and perioperative outcomes. Spine 2014; 39: E111-122.

6. Sinikallio S, Aalto T, Airaksinen O, Herno A, Kröger H, Savolainen S, et al. Depressive symptoms predict postoperative disability among patients with lumbar spinal stenosis: a two-year prospective study comparing two age groups. Disabil Rehabil. 2009; 30: 415-422.

7. Levy HI, Hanscom B, Boden SD. Three-question depression screener used for lumbar disc herniations and spinal stenosis. Spine 2002; 27: 1232-1237.
8. Eaton W, Smith C, Ybarra M, Muntaner C, Tien A. Center for Epidemiologic Studies Depression Scale: review and revision (CESD and CESD-R). In Maruish M (ed) The Use of Psychological Testing for Treatment Planning and Outcomes Assessment. Mahwah, NJ: Lawrence Erlbaum, 2004: 363-367.

9. Van Dam NT, Earleywine M. Validation of the Center for Epidemiologic Studies Depression Scale-Revised (CESD-R): Pragmatic depression assessment in the general population. Psychiatry Res. 2011; 186: 128-132.

10. Faulstich ME, Carey MP, Ruggiero L, Enyart P, Gresham F. Assessment of depression in childhood and adolescence: an evaluation of the Center for Epidemiological Studies Depression Scale for Children (CES-DC). Am J Psychiatry. 1986; 143: 1024-1027.

11. Lewinsohn PM, Seeley JR, Roberts RE, Allen NB.. Center for Epidemiologic Studies Depression Scale (CES-D) as a screening instrument for depression among community-residing older adults. Psychol Aging. 1997; 12: 277-287.

12. Radloff LS. The use of the Center for Epidemiologic Studies Depression Scale in adolescents and young adults. J Youth Adolesc. 1991; 20: 149-166.

13. Radloff LS. The CES-D Scale A Self-Report Depression Scale for Research in the General Population. Appl Psychol Meas. 1977; 1: 385-401.

14. Ducat L, Philipson LH, Anderson BJ. The Mental Health Comorbidities of Diabetes. JAMA. 2014; 312: 691-692.

15. Kanner AM. Depression in epilepsy: prevalence, clinical semiology, pathogenic mechanisms, and treatment. Biol Psychiatry. 2003; 54: 388-398.

16. Mullish BH, Kabir MS, Thursz MR, Dhar A. Review article: depression and the use of antidepressants in patients with chronic liver disease or liver transplantation. Aliment Pharmacol Ther. 2014; 40: 880-892.

17. Ni Mhaolain AM, Butler JS, Magill PF, Wood AE, Sheehan J. The increased need for liaison psychiatry in surgical patients due to the high prevalence of undiagnosed anxiety and depression. Ir J Med Sci. 2008; 177: 211-215.

18. Pence BW. The impact of mental health and traumatic life experiences on antiretroviral treatment outcomes for people living with HIVIAIDS. J Antimicrob Chemother. 2009; 63: 636-640.

19. Herr KA, Mobily PR, Smith C. Depression and the experience of chronic back pain: a study of related variables and age differences. Clin J Pain 1993; 9: 104-114.

20. Urban-Baeza A, Zárate-Kalfópulos B, Romero-Vargas S, Obil-Chavarría C, Brenes-Rojas L, Reyes-Sánchez A. Influence of depression symptoms on patient expectations and clinical outcomes in the surgical management of spinal stenosis. J Neurosurg Spine. 2015; 22: 75-79.

21. Polatin PB, Kinney RK, Gatchel RJ, Lillo E, Mayer TG. Psychiatric illness and chronic low-back pain. The mind and the spine--which goes first?. Spine 1993; 18: 66-71.

22. Geisser ME, Roth RS, Robinson ME. Assessing depression among persons with chronic pain using the Center for Epidemiological Studies-Depression Scale and the Beck Depression Inventory: a comparative analysis. Clin J Pain 1997; 13: 163-170.

23. Gomez R, McLaren S. The center for epidemiologic studies depression scale: support for a bifactor model with a dominant general factor and a specific factor for positive affect. Assessment. 2015; 22: 351-360.

24. IsHak WW, Greenberg JM, Balayan K, Kapitanski N, Jeffrey J, Fathy H, et al. Quality of life: the ultimate outcome measure of interventions in major depressive disorder. Harv Rev Psychiatry. 2011; 19: 229-239.
Ann Depress Anxiety - Volume 5 Issue 1 - 2018 ISSN : 2381-8883 | www.austinpublishing group.com Dawkins et al. (C) All rights are reserved
Citation: Menendez JY, Dawkins RL, Walters BC, Shelton RC, Markert JM and Pritchard PR. The Prevalence of Undiagnosed Depression in Patients with Spinal Pathology. Ann Depress Anxiety. 2018; 5(1): 1091. 